\title{
EFFECTS OF STATISTICAL ROUGHNESS ON THE PROPAGATION OF ELECTROMAGNETIC FIELDS IN A CIRCULAR WAVEGUIDE
}

\author{
L. Palumbo*, E. Di Liberto, F. Frezza** \\ *Dip. Energetica-Università di Roma "La Sapienza" \\ **Dip. di Ingegneria Elettronica-Università di Roma "La Sapienza"
}

\begin{abstract}
The Green's function method (GFM) is used to describe the effects of a statistical roughness on the propagation of electromagnetic fields in a circular waveguide. The GFM method, applied to a scalar Helmholtz equation with perturbed boundary conditions, allows us to treat multiple scattering of the field. Theoretical results for azimuthal and longitudinal roughness are reported, and the phase velocity of the field is discussed in both cases.
\end{abstract}

\section{INTRODUCTION}

In the design of future Free Electron Lasers and Linacs very short bunches of high intensity, small emittance and small energy spread are planned to be used. In order to keep limited the degradation of beam features, evaluation of induced wakefields acquires considerable importance: in Ref. [1] it has been remarked, as regards accelerators working with short bunches, that the roughness of beam pipe surface might excite parasitic fields increasing emittance and energy spread.

Several simplified models have been developed to evaluate these effects, assuming irregularities either as small bumps with simple shape and random distribution on a smooth surface [1] or as a periodic corrugation of beam tube [2]; a further model [1] describes the roughness, into a local cartesian reference frame, as a random function $y=h(x, z)$ of surface coordinates $x$ and $z$ and longitudinal impedance is calculated depending on statistical properties of the process $h$, assuming small local derivatives (smallangle approximation, $|\nabla h| \ll 1)$.

In this paper we investigate the effects of surface roughness on an electromagnetic wave travelling in a circular waveguide whose boundary is given by the 2-D equation $a+\tilde{\rho}(\varphi, z)$. It should be noted that an electromagnetic wave incident on a rough surface is subjected to diffraction phenomena which can be divided into two groups according to the number of total reflections: the first group comprises processes like incidence on sea surface or irregular ground, where the wave undergoes a single act of scattering and only slight distorsions of the field are produced [3]; the second group is made by the phenomena that take place in domains bounded by two or more scattering surfaces, like in rough resonant cavities or waveguides, where a field undergoes multiple scattering along propagation path and a simple pertubation theory is not very fruitful to describe it correctly. The GFM allows us to treat distributed summation of waves propagating in a waveg- uide with randomly rough shape, approximating an homogeneous boundary condition on the perturbed surface by an inhomogeneous condition calculated on the average, i.e. ideal, surface. Being $\tilde{\rho}(\varphi, z)$ a random function of transverse coordinates, we calculate, depending on its statistical features (variance and correlation function), the phase velocity shift for the electromagnetic field: within the range of validity of our approximations, no synchronous fields, propagating along the waveguide with $v_{p h}=c$, emerge for a finite value of the frequency $f=\omega / 2 \pi$.

\section{THE GREEN'S FUNCTION METHOD}

\subsection{General Description}

Let us consider the function $G\left(\mathbf{r}, \mathbf{r}^{\prime}\right)$ solution of three dimensional Helmholtz equation

$$
\nabla^{2} G\left(\mathbf{r}, \mathbf{r}^{\prime}\right)+k^{2} G\left(\mathbf{r}, \mathbf{r}^{\prime}\right)=\delta\left(\mathbf{r}-\mathbf{r}^{\prime}\right)
$$

with the mixed boundary condition (BC)

$$
\frac{\partial G\left(\mathbf{r}, \mathbf{r}^{\prime}\right)}{\partial n_{p}}+\left.\eta_{o} G\left(\mathbf{r}, \mathbf{r}^{\prime}\right)\right|_{\mathbf{r} \in S_{p}}=0
$$

where $\eta_{o}$ is an integro-differential operator and $\mathbf{n}_{p}$ is a unit vector normal to the surface $S_{p}$, described by the radius vector

$$
\mathbf{r}=\mathbf{r}_{s}+\mathbf{n}\left(\mathbf{r}_{s}\right) \zeta\left(\mathbf{r}_{s}\right)
$$

where $\mathbf{r}_{s}$ lies on the ideal surface $S$ that undergoes shape deviations given by the random function $\zeta\left(\mathbf{r}_{s}\right)$. In the following we will consider as known the function $G_{o}\left(\mathbf{r}, \mathbf{r}^{\prime}\right)$, that is the solution of Helmholtz problem when no perturbation is present.

Assuming that $\left|\nabla_{t} \zeta\left(\mathbf{r}_{s}\right)\right| \ll 1$, with $\nabla_{t}=\nabla-\mathbf{n}(\nabla \cdot \mathbf{n})$, and substituting (3) into (2), the BC can be transferred on the ideal surface $S$ by means of an expansion in the perturbation parameter $\zeta$, retaining only first order terms

$$
\frac{\partial G}{\partial n}+\eta_{o} G-\nabla_{t} \zeta \cdot \nabla_{t} G+\zeta \frac{\partial^{2} G}{\partial n^{2}}+\left.\eta_{o} \zeta \frac{\partial G}{\partial n}\right|_{\mathbf{r} \in S}=0
$$

This equation is known as approximated or effective boundary condition (EBC) that can be shortly written as $\left[\partial G / \partial n+\eta_{o} G+\hat{V} G\right]_{S}=0$, that is an unperturbed condition corrected by a perturbation term proportional to the random 
parameter $\zeta$. Using Green's theorem

$$
\begin{aligned}
G\left(\mathbf{r}, \mathbf{r}^{\prime}\right) & =G_{o}\left(\mathbf{r}, \mathbf{r}^{\prime}\right)+\int_{S}\left[\frac{\partial G_{o}}{\partial n} G+G_{o} \frac{\partial G}{\partial n}\right] d S_{s}= \\
= & G_{o}\left(\mathbf{r}, \mathbf{r}^{\prime}\right)+\int_{S} G_{o}\left(\mathbf{r}, \mathbf{r}_{s}\right) \hat{V}\left(\mathbf{r}_{s}\right) G\left(\mathbf{r}_{s}, \mathbf{r}^{\prime}\right) d S_{s}
\end{aligned}
$$

we obtain an integral equation for $G\left(\mathbf{r}, \mathbf{r}^{\prime}\right)$, depending on the random parameter $\hat{V}(\zeta)$. It can be shown that even the average Green's function $\mathcal{G}\left(\mathbf{r}, \mathbf{r}^{\prime}\right)=<G\left(\mathbf{r}, \mathbf{r}^{\prime}\right)>$ is solution of an integral equation

$$
\mathcal{G}=G_{o}+\iint_{S} G_{o}\left(\mathbf{r}, \mathbf{r}_{s_{1}}\right) \mathcal{M}\left(\mathbf{r}_{s_{1}}, \mathbf{r}_{s_{2}}\right) \mathcal{G}\left(\mathbf{r}_{s_{2}}, \mathbf{r}^{\prime}\right) d S_{s_{1}} d S_{s_{2}}
$$

where $\mathbf{r}_{s_{i}} \in S$, that is called Dyson equation; the mass operator function $\mathcal{M}$, introducing dependence on random function $\zeta$, can be expressed as an infinite series, performing a multiple iteration of eq. (6) ${ }^{1}$. If one let $\mathbf{r}$ approach $S$, because of the properties of unperturbed Green's function, $\mathcal{G}\left(\mathbf{r}, \mathbf{r}^{\prime}\right)$ verifies a non-local $\mathrm{BC}$ given by

$$
\left.\left(\frac{\partial}{\partial n}+\eta_{o}\right) \mathcal{G}\left(\mathbf{r}, \mathbf{r}^{\prime}\right)\right|_{S}=\int_{S} \mathcal{M}\left(\mathbf{r}_{s}, \mathbf{r}_{s_{1}}\right) \mathcal{G}\left(\mathbf{r}_{s_{1}}, \mathbf{r}^{\prime}\right) d S_{s_{1}}
$$

\section{CIRCULAR WAVEGUIDE WITH RANDOM ROUGHNESS}

In a cylindrical system of coordinates $\{\rho, \varphi, z\}$ a set of solutions corresponding to $\mathrm{TM}^{(z)}$ e.m. waves can always be derived from the non-null longitudinal component of electric field $E_{z}$, considered as potential function, that is solution of

$$
\nabla^{2} E_{z}+k^{2} E_{z}=0
$$

with the further condition $\mathbf{n} \times\left.\boldsymbol{E}\right|_{S}=0$ when a bounding metallic surface $S$ is present. If this surface can be described as $a+\tilde{\rho}(\varphi, z)$, then its unit vector is $\mathbf{n}_{p} \cong$ $\left[1,-\frac{\partial \tilde{\rho}}{\partial \varphi},-\frac{\partial \tilde{\rho}}{\partial z}\right]$ and the vanishing condition for $\boldsymbol{E}$ becomes

$$
\frac{\partial \tilde{\rho}}{\partial z} E_{\rho}+E_{z}=0
$$

Performing separated analysis for azimuthal and longitudinal corrugations, we have made differences stand out as regards eigenvalue spectrum, pointing out typical features of both cases.

\subsection{Azimuthal Roughness}

If the boundary surface of the waveguide is given by $a+$ $\tilde{\rho}(\varphi)$, simple geometrical considerations allow us to treat a

\footnotetext{
${ }^{1}$ Actually the GFM was originally introduced to describe wave propagation into 3-D random media, employing Feynman diagram technique: the treatment shown in this paper derives from an extension to the case of boundary perturbations [4].
}

bidimensional Helmholtz equation in the transverse plane, assuming for all solutions an exponential dependence ${ }^{2}$ on $z$ like $e^{-\jmath k_{z} z}$. Since $\tilde{\rho}$ varies only with azimuthal coordinate, equation (9) entails $E_{z}=0$ on $S_{p}$. The technique presented in previous section has been applied to a Green's problem equivalent to equations (8)-(9).

The EBC for azimuthal perturbations appears as

$$
G\left(\rho, \varphi, \rho^{\prime}, \varphi^{\prime}\right)+\left.\frac{\partial G\left(\rho, \varphi, \rho^{\prime}, \varphi^{\prime}\right)}{\partial \rho} \tilde{\rho}(\varphi)\right|_{\rho=a}=0
$$

and the Green's theorem for bidimensional domains leads to the integral Dyson equation

$$
\begin{gathered}
\mathcal{G}\left(\mathbf{r}_{t}, \mathbf{r}_{t}^{\prime}\right)=G_{o}\left(\mathbf{r}_{t}, \mathbf{r}_{t}^{\prime}\right)+\iint_{S} \frac{\partial G_{o}\left(\mathbf{r}_{t}, \mathbf{r}_{t_{s}}\right)}{\partial n_{s}} M\left(\mathbf{r}_{t_{s}}, \mathbf{r}_{t_{s}^{\prime}}\right) \times \\
\times \frac{\partial \mathcal{G}\left(\mathbf{r}_{t_{s}}, \mathbf{r}_{t}^{\prime}\right)}{\partial n_{s}^{\prime}} d \mathbf{r}_{t_{s}} d \mathbf{r}_{t_{s}}^{\prime} \quad \mathbf{r}_{t_{s}}=(a, \varphi)
\end{gathered}
$$

In this situation the non-local boundary condition is

$$
\left.\mathcal{G}\right|_{\rho=a}=\left.\int_{S} M\left(\varphi, \varphi^{\prime \prime}\right) \frac{\partial \mathcal{G}\left(\rho^{\prime \prime}, \varphi^{\prime \prime}, \rho^{\prime}, \varphi^{\prime}\right)}{\partial \rho^{\prime \prime}}\right|_{\rho^{\prime \prime}=a} ^{a d \varphi^{\prime \prime}}
$$

because of the properties of unperturbed Green's function for Dirichlet conditions.

If one assumes $\tilde{\rho}$ as a statistically uniform process, it is uneffected by waveguide rotations and the Green's function can be still considered as depending only on $\left(\varphi-\varphi^{\prime}\right)$. Developing both members of (12) in Fourier series, a mixed boundary condition at $\rho=a$ emerges for harmonic components $\mathcal{G}_{n}\left(\rho, \rho^{\prime}\right)$ of average Green function

$$
\mathcal{G}_{n}\left(a, \rho^{\prime}\right)=\left.2 \pi a M_{n} \frac{\partial \mathcal{G}_{n}\left(\rho, \rho^{\prime}\right)}{\partial \rho}\right|_{\rho=a}
$$

Being $\mathcal{G}_{n}$ solutions of a Bessel differential equation ${ }^{3}$ verifying condition (13), one obtains an expression for $\mathcal{G}_{n}\left(\rho, \rho^{\prime}\right)$ [5] and finally a perturbed dispersion equation of the form

$$
J_{n}\left(k_{\rho} a\right)-2 \pi a k_{\rho} M_{n}\left(k_{z}\right) J_{n}^{\prime}\left(k_{\rho} a\right)=0
$$

An approximated evaluation of transverse eigenvalue shift is possible, assuming $\left|\delta k_{\rho}\right|$ much smaller than the distance between two adjacent roots and thus solving (14) as a perturbation of $J_{n}\left(k_{\rho} a\right)=0$, finally obtaining

$$
\delta k_{\rho} \cong 2 \pi k_{\rho} M_{n}\left(k_{z}\right)
$$

The random process $\tilde{\rho}$ appears into Fourier components $M_{n}$ of the function $M\left(\varphi, \varphi^{\prime}\right)$; if $\sigma$ is the variance of $\tilde{\rho}$ and $W\left(\varphi-\varphi^{\prime}\right)=\sigma^{-2}<\tilde{\rho}(\varphi) \tilde{\rho}\left(\varphi^{\prime}\right)>$ is its correlation coefficient, then, neglecting higher order terms proportional to

\footnotetext{
${ }^{2}$ Let us remind that this is actually equivalent to perform a Fourier transform.

${ }^{3}$ In fact the operations of differentiation and averaging, because of their linearity, are interchangeable.
} 
$(\sigma / a)^{4}$ in the infinite series defining mass operator (Buorret approximation [4], see Sect. 2) it follows:

$$
\left.M\left(\varphi, \varphi^{\prime}\right) \approx \sigma^{2} W\left(\varphi-\varphi^{\prime}\right) \frac{\partial^{2} G_{o}\left(\rho, \varphi, \rho^{\prime}, \varphi^{\prime}\right)}{\partial \rho \partial \rho^{\prime}}\right|_{\rho=\rho^{\prime}=a}
$$

and then, from eq. (15)

$$
\delta k_{\rho_{n, m}}=2 \pi k_{\rho_{n, m}} \frac{\sigma^{2}}{\pi a^{4}} \sum_{p=0}^{+\infty} \sum_{q=1}^{+\infty} \frac{\epsilon_{p}}{2} \xi_{p, q}^{2} \frac{W_{n-p}+W_{n+p}}{k_{z_{p, q}}^{2}-k_{z_{n, m}}^{2}}
$$

where $\epsilon_{p}$ is the Neumann symbol, $\xi_{p, q}$ is the q-th zero of Bessel function $J_{p}(x), k_{z_{p, q}}=\left[k^{2}-\left(\xi_{p, q} / a\right)^{2}\right]^{1 / 2}$, and $W_{i}$ represents the i-th Fourier harmonic of the correlation coefficient $W\left(\varphi, \varphi^{\prime}\right)$. In eq. (17) the term with index $\{n, m\}$ shows a null denominator as consequence of previous approximations. The resonance can be avoided performing a more accurate evaluation that finally leads, retaining only dominant term, to

$$
\delta k_{z_{n, m}}=\left(\frac{d k_{z}}{d k_{\rho}}\right) \delta k_{\rho_{n, m}}=\left(\frac{\sigma}{a}\right) \frac{k_{\rho_{n, m}}^{2}}{k_{z_{n, m}}} \sqrt{W_{o}}
$$

as regards propagation constant $k_{z}=\left[k^{2}-k_{\rho}^{2}\right]^{1 / 2}$. In Fig. 1 (a) the normalized shift for $\mathrm{TM}_{0,1}$ is plotted versus normalized frequency, for different values of $k l$, where $l$ is the correlation length, assuming for $\tilde{\rho}$ a gaussian statistics.

The decreasing of phase velocity determined by eq. (18) appears to be very small and, within the range of validity of our approximations, no synchronuos field seems to be excited.

\subsection{Longitudinal Roughness}

If the boundary surface of the waveguide is now given by $a+\tilde{\rho}(z)$, it is no longer possible to separate longitudinal propagation from transverse resonance: anyway one can still consider a bidimensional problem, now in the $(\rho, z)$ plane, with the condition $E_{z}=0$ on the rough boundary, provided that the first term of eq. (9) might be neglectable in case of small perturbations with small gradient. Calculations absolutely similar to those of previous section again lead to the perturbed dispersion equation (14). The difference is in the form of mass operator transform, appearing now as a continuous convolution

$$
M_{n}\left(k_{z}\right)=\frac{\sigma^{2}}{4 \pi^{2}} \frac{1}{\pi a^{4}} \sum_{q=1}^{+\infty} \xi_{n, q}^{2} \int_{-\infty}^{+\infty} \frac{\tilde{W}\left(k_{z}-k_{z}^{\prime}\right)}{k_{z_{n}, q}^{2}-k_{z}^{\prime 2}} d k_{z}^{\prime}
$$

where $\tilde{W}\left(k_{z}\right)$ is the Fourier transform of the correlation coefficient $W\left(z-z^{\prime}\right)$. In order to determine eigenvalue shift, it is necessary to utilize the radiation principle [4] in the solution of the intergral present in eq. (19). It appears that now $\delta k_{z_{n, m}}$ holds real and imaginary part

$$
\begin{aligned}
& \Re\left[\delta k_{z_{n, m}}\right]=\frac{k_{\rho_{n, m}}^{2}}{k_{z_{n, m}}} \frac{\sigma^{2}}{2 \pi a^{4}} \sum_{q=Q(n)+1}^{+\infty} \xi_{n, q}^{2} \frac{\tilde{W}\left(k_{z_{n, m}}+\jmath \alpha_{z_{n, q}}\right)}{\alpha_{z_{n, q}}} \\
& \Im\left[\delta k_{z_{n, m}}\right]=-\frac{k_{\rho_{n, m}}^{2}}{k_{z_{n, m}}} \frac{\sigma^{2}}{2 \pi a^{4}} \sum_{q=1}^{Q(n)} \xi_{n, q}^{2} \frac{F(\tilde{W})}{\beta_{z_{n, q}}}
\end{aligned}
$$

where $Q$ verifies $\xi_{n, 1}<a k<\xi_{n+1,1}$ and $\xi_{n, Q}<a k<\xi_{n, Q+1}$, and $F(\tilde{W})=\left[\tilde{W}\left(k_{z_{n, m}}-k_{z_{n, q}}\right)+\tilde{W}\left(k_{z_{n, m}}+k_{z_{n, q}}\right)\right]$.

The incoherent scattering into other modes that can propagate above cut-off along the waveguide (both in forward and in backward direction; see $F[\tilde{W}]$ ) causes an attenuation given by eq. $(20 \mathrm{~b})$, while the excited modes below cut-off decrease the phase velocity according to eq. (20 a). In Fig. 1 (b) the normalized attenuation for $\mathrm{TM}_{0,1}$ is plotted versus normalized frequency, showing resonance peaks by different cut-offs: in fact an eigenwave propagating along the waveguide with a steep angle of incidence, being frequently reflected, is more influenced by roughness and thus the effect of perturbation is amplified.

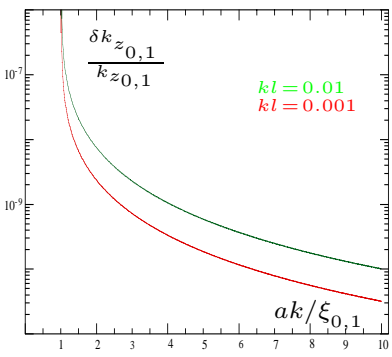

(a)

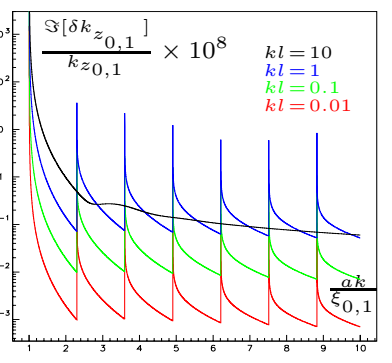

(b)
Figure 1: Normalized shift vs. normalized frequencies: azimuthal (a), and longitudinal (b) roughness.

\section{CONCLUSIONS}

The GFM allows us to consider multiple scattering of e.m. waves into a randomly rough waveguide, determining, as regards $\mathrm{TM}_{n, m}$ modes of the structure, the actual shift for propagation constant $\delta k_{z}$ for azimuthal and longitudinal corrugations. In both cases a decreasing in phase velocity emerges, depending on statistical features of random perturbation, which, within the range of validity of our approximations, does not appear sufficient to excite slow waves. For longitudinal roughness, an attenuation term has been found taking into account incoherent scattering power losses.

\section{REFERENCES}

[1] G. V. Stupakov, K. L. F. Bane "Wake of a rough beam wall surface", SLAC-PUB, 8023, Dec.1998.

[2] M. Angelici, A. Mostacci, L. Palumbo, F. Ruggiero, S. Ugoli "Wake Field Effects due to Surface Roughness", VIII International Workshop on Linear Colliders, Frascati, 1999.

[3] I. Fuks, F. Bass, V. Freilikher "Propagation in Statistically Irregular Waveguides. Part I: Average Field", IEEE Transactions on Antennas and Propagation, vol.22, March 1974.

[4] I. Fuks, F. Bass "Wave Scattering from Statistically Rough Surfaces", Pergamon Press, Oxford, 1979.

[5] E. Di Liberto, "Il metodo della funzione di Green per la valutazione degli effetti delle rugosità statistiche sulla propagazione di modi in guida" (in italian), laurea thesis 1999. 